\title{
Transport processes in $\mathrm{YBa}_{2} \mathrm{Cu}_{3} \mathrm{O}_{x} / \mathrm{PrBa}_{2} \mathrm{Cu}_{3} \mathrm{O}_{x} / \mathrm{YBa}_{2} \mathrm{Cu}_{3} \mathrm{O}_{x}$ ramp type Josephson junctions
}

\author{
Yu.M. Boguslavskij ', J. Gao, A.J.H.M. Rijnders, D. Terpstra, G.J. Gerritsma and H. Rogalla \\ Department of Applied Physics, University of Twente, PO Box 217.7500 AE Enschede, Netherlands
}

Received 30 January 1992

Revised manuscript received 3 March 1992

\begin{abstract}
A study of the $\mathrm{YBCO} / \mathrm{PBCO} / \mathrm{YBCO}$ ramp junctions with and without PBCO barrier shows that the Josephson and normal state behavior of these structures are determined by the thickness of the PBCO barrier and its nature. The boundary resistance and depression of the $\mathrm{YBCO}$ superconducting parameters near the interface do not strongly affect the junction characteristic. For thicknesses of 8 to $20 \mathrm{~nm}$ of the PBCO barrier the Josephson coupling is established through the high resistive barrier and the behavior of the junctions is better described by a SNINS model than by a SNS weak link model. Proximity effect, resonant tunneling and strong pair breaking mechanisms are discussed to explain the experimental characteristics. Good agreement with the experimental dependence of the $I_{\mathrm{c}} R_{\mathrm{n}}$ product on the temperature and on the PBCO barrier thickness was obtained if a strong pair breaking mechanisms in the barrier is taken into account.
\end{abstract}

\section{Introduction}

The operation of superconducting devices at relatively high temperatures $T \sim 40-77 \mathrm{~K}$ puts some requirements on their parameters. The absolute value of the critical current $I_{\mathrm{c}}$ must be several times higher than the thermal noise level $I_{\mathrm{th}}=2 \pi k_{\mathrm{B}} T / \Phi_{0}$. For nonhysteretic operation the dimensionless parameter $\beta_{j}=\left(\Phi_{0} / 2 \pi I_{\mathrm{c}} C R_{\mathrm{n}}^{2}\right)^{1 / 2}$ must be $\beta_{j} \geqslant 1$. Here $C$ is the junction capacitance and $R_{\mathrm{n}}$ is the normal state resistance. For junctions or SQUIDs in the optimal operating regime the maximum achievable voltage modulation in an applied magnetic field is $\Delta V_{\mathrm{m}} \sim I_{\mathrm{c}} R_{\mathrm{n}} / 2$ and the maximum flux responsity is $\partial V / \partial \Phi \sim I_{\mathrm{c}} R_{\mathrm{n}} / \Phi_{0}$. The resistance $R_{\mathrm{n}}$ of the superconducting devices should match the resistance of other elements of the circuit.

These requirements mainly concern the achievable $I_{\mathrm{r}} R_{\mathrm{n}}$ product, its temperature dependence and the $I_{c}\left(R_{n}\right)$ dependence. These parameters and dependences differ for all proposed high- $T_{\mathrm{c}}$ junctions, such as grain boundary junctions [1-5], weak link struc-

1 On leave from the Institute of Metal Physics, $252142 \mathrm{Kiev}$, Ukraine. tures with a low ohmic noble metal $[6,7]$, multilayer epitaxial structures [8-10], junctions with passivation of the $\mathrm{YBa}_{2} \mathrm{Cu}_{3} \mathrm{O}_{x}$ surface in a plasma [11] or with an N-YBCO layer deposited at low temperatures [12]. The Josephson coupling between the superconducting electrodes is established in different ways: through the high angle grain boundary, through the layer of low ohmic $\mathrm{Ag}$ or $\mathrm{Ag} / \mathrm{Au}$, high ohmic $\mathrm{PrBa}_{2} \mathrm{Cu}_{3} \mathrm{O}_{x}$ or $\mathrm{Nb}$-doped $\mathrm{SrTiO}_{3}$. Although the Josephson effect via response to magnetic field or microwave irradiation was observed in most of these structures, the nature of the barrier often remains unclear. The influence of the barrier nature and interface quality on the Josephson and normal state junction behavior is the key problem for the improvement of the high- $T_{\mathrm{c}}$ junction parameters.

Atomically sharp boundaries in SNS structures and low carrier scattering at the interfaces seem to be ensured in multilayer structures having a similar crystal structure. $\operatorname{PrBa}_{2} \mathrm{Cu}_{3} \mathrm{O}_{x}$ (PBCO) is a convenient semiconductor-like material which is isomorphic to the high- $T_{\mathrm{c}}$ superconductor (HTS) $\mathrm{YBa}_{2} \mathrm{Cu}_{3} \mathrm{O}_{x}$ ( $\mathrm{YBCO}$ ) and can be used as a barrier material. Our study of the $\mathrm{YBCO} / \mathrm{PBCO} / \mathrm{YBCO}$ multilayers and Josephson ramp junctions [10,13-15] shows high 
quality crystal structures and interlayer boundaries as well as reproducible characteristics, including $I_{\mathrm{c}} R_{\mathrm{n}}$ products up to $8 \mathrm{mV}$ at $4.2 \mathrm{~K}$, clear Josephson behavior and scaling of the junction parameters with the junction area. For better understanding of the Josephson effect in the YBCO/PBCO/YBCO ramp structures a more detailed study of the mechanisms of the current passage through this barrier layer is needed.

In this article the general characteristics of the $\mathrm{YBCO} / \mathrm{PBCO} / \mathrm{YBCO}$ ramp (low angle edge) junctions with different barrier thicknesses are studied. The influence of the boundary resistance between different epitaxial layers is estimated from the study of the junctions without a PBCO barrier layer. Different mechanisms of current passage through the PBCO barrier are discussed in order to explain the observed junction behavior and the reduction of the $I_{\mathrm{c}} R_{\mathrm{n}}$ products in the $\mathrm{YBCO} / \mathrm{PBCO} / \mathrm{YBCO}$ junctions.

\section{Characterization of the $\mathrm{YBCO} / \mathrm{PBCO} / \mathrm{YBCO}$ ramp junctions}

We have previously reported on the fabrication process of high quality epitaxial high- $T_{\mathrm{c}}$ ramp type $\mathrm{YBCO} / \mathrm{PBCO} / \mathrm{YBCO}$ Josephson junctions $[10,15]$. Briefly, all layers were deposited by a modified offaxis sputtering technique. The base YBCO electrode was covered by a thick ( $>250 \mathrm{~nm}$ ) PBCO layer to avoid electrical shunting of the superconducting electrodes in the overlap area. The edge of the junction was created by low angle $\mathrm{Ar}$ ion beam etching. The thin PBCO barrier layer and the YBCO top layer were deposited on the edge of the base electrode after additional cleaning of the edge surface by a low power Ar ion beam. The desired junction structure was defined by photolithographic patterning and ion beam etching. The thickness of the layers was set by the sputtering time of the well-calibrated and reproducible deposition process and was checked by a step profilometer.

The junctions cross section area was determined as $A=w \times d / \sin \vartheta$, where $w$ is the junction width, $d$ is the thickness of the base electrode and $\vartheta$ is the angle of the junction edge. This geometrical value is close to the value of the junction area determined from the modulation of the critical current in an ap- plied magnetic field, as we reported earlier [14]. The normal state resistance $R_{\mathrm{n}}$ was determined from the linear part of the current-voltage characteristics at high voltages $V \sim 10-30 \mathrm{mV}$. The accuracy of this method was checked by suppression of the critical current by microwave irradiation. The slope of the $I-V$ characteristics (IVC) between the microwaveinduced current steps agrees well with the slope of the IVC at higher voltages $V \sim 10-30 \mathrm{mV}$. The estimated accuracy of the critical current density $J_{\mathrm{c}}$ and $R_{\mathrm{n}} A$ product values is about 20 percent.

Characterization of the $\mathrm{YBCO} / \mathrm{PBCO} / \mathrm{YBCO}$ junctions with different areas and barrier thicknesses $[14,15]$ shows clear Josephson behavior in an applied magnetic field and under microwave irradiation as well as the uniformity of the junction parameters and their scaling with junction area. The fabrication process is controllable and reproducible for this type of all high- $T_{\mathrm{c}}$ junctions.

The coherence length of the PBCO barrier can be estimated from the dependence of the critical current density $J_{c}$ on the barrier thickness $L$. To a good approximation $J_{\mathrm{c}}$ decreases exponentially as $L$ increases from $L \geqslant 10 \mathrm{~nm}$. Using the expression [16]

$\ln J_{\mathrm{c}} \sim \ln J_{\mathrm{c} 0}-L / \xi_{\mathrm{n}}$,

we have estimated the effective coherence length to be $\xi_{n} \sim 5-8 \mathrm{~nm}$, as we reported and discussed earlier $[14,15]$.

The Josephson and normal state junction characteristics depend strongly on the interlayer boundary resistance, the thickness of the PBCO barrier layer and the physical nature of the PBCO barrier material. First we will consider the interlayer boundary resistance. The similar crystal structure of the YBCO and $\mathrm{PBCO}$ layers gives the opportunity to produce high quality heteroepitaxial $\mathrm{YBCO} / \mathrm{PBCO} / \mathrm{YBCO}$ multilayers. The main question concerns the quality of the edge of the base electrode after final low energy ion beam cleaning before deposition of the PBCO barrier layer and top YBCO electrode.

Junctions without a PBCO barrier have been studied to estimate the influence of the ex-situ fabrication process and the quality of the edge surface on the boundary resistance $R_{\mathrm{b}}$ value and on the depression of the YBCO superconducting parameters near the interface. These junctions have been prepared under similar multilayer process conditions, includ- 
ing ion beam etching and edge surface cleaning parameters. At $T>T_{\mathrm{c}}$ the measured resistance of the junctions is the sum of the series connected base and top YBCO electrodes resistances. The critical temperature $T_{\text {co }}$ of the junctions is equal to the critical temperature of the top electrode $T_{\mathrm{c} 0}=87.5 \mathrm{~K}$ (for the base electrode $T_{\mathrm{c} 0}$ is $88 \mathrm{~K}$ ). Figure 1 shows the temperature dependence of the critical current density $J_{c}$ of the YBCO/YBCO junction (curve A) and of the $\mathrm{YBCO} / \mathrm{PBCO} / \mathrm{YBCO}$ junction with a barrier thickness of $8 \mathrm{~nm}$. The $J_{\mathrm{c}}(T)$ dependence of the $\mathrm{YBCO} / \mathrm{YBCO}$ junctions is $J_{\mathrm{c}} \sim\left(T_{\mathrm{c}}-T\right)^{2}$, which is typical for junctions with a thin PBCO barrier [10]. However, the absolute value of the critical current density $J_{\mathrm{c}}(T)$ is several orders of magnitude higher than that of the junctions with a PBCO barrier at the same temperatures, as fig. 1 shows. The critical current $I_{\mathrm{c}}$ scales with the width of the junction in the range of $w=5-40 \mu \mathrm{m}$.

The upper limit of the interface boundary resistance for such junctions is estimated to be $R_{\mathrm{b}} \leqslant 3-$ $7 \times 10^{-9} \Omega \mathrm{cm}^{2}$. This value is negligible in comparison with typical values of $R_{\mathrm{b}}$ of the junction with a PBCO barrier thickness $L=6-20 \mathrm{~nm}$.

These results show that the damage of the surface due to the fabrication process used, has a very small effect on the boundary resistances and the superconducting properties of the junctions. All YBCO and

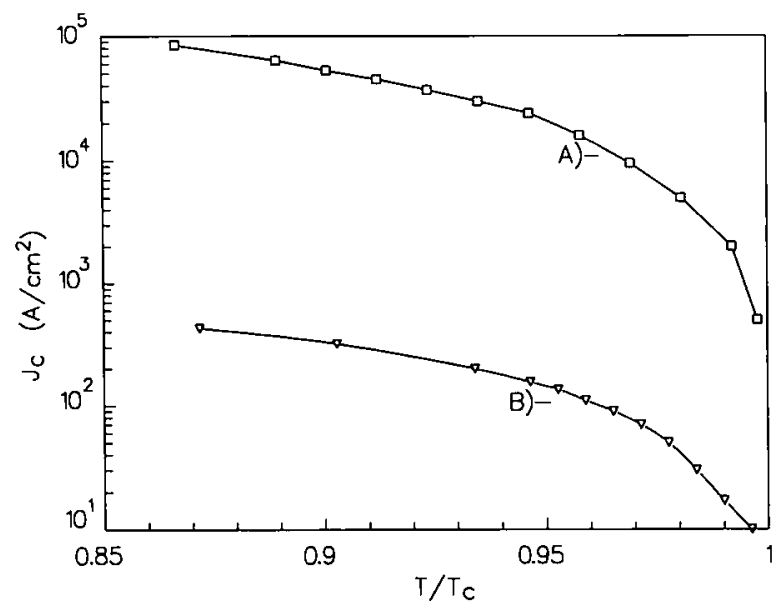

Fig. 1. Temperature dependence of the critical current density $J_{c}$ of the $\mathrm{YBCO} / \mathrm{YBCO}$ junctions without $\mathrm{PBCO}$ barrier (curve A) and of the junctions with a PBCO barrier thickness of $8 \mathrm{~nm}$ (curve B).
PBCO layers are deposited in analogous conditions. YBCO and PBCO materials have a similar crystal structure, a similar effect of oxygen on the structural ortho-tetra phase transition, etc. [17]. Thus, the boundary resistance $R_{\mathrm{b}}$ between the $\mathrm{YBCO}$ and PBCO layers is expected to be in the same range $R_{\mathrm{b}} \leqslant 3-7 \times 10^{-9} \Omega \mathrm{cm}^{2}$. We will use these results for the analysis of the effect of the PBCO barrier in the $\mathrm{YBCO} / \mathrm{PBCO} / \mathrm{YBCO}$ structure.

The strength of the Josephson coupling between the superconducting electrodes depends on the nature of the barrier and the quality of the superconducting electrodes in the near barrier region. In the different types of proposed Josephson structures the mechanisms of the current passage may be different. A comparison of the dependences of the critical current density $J_{\mathrm{c}}$ on the junction specific resistance $R_{\mathrm{n}} A$ in the different types of the junctions can clarify the effect of the PBCO barrier.

Figure 2 shows the experimental data for the $J_{\mathrm{c}}\left(R_{\mathrm{n}} A\right)$ dependence of the $\mathrm{YBCO} / \mathrm{PBCO} / \mathrm{YBCO}$ ramp junctions (marked by a triangle, fitting curve $A$ ), of the grain boundary junctions (GBJ) on a $\mathrm{SrTiO}_{3}$ bicrystal (from ref. [1], curve B), of the intergrain weak links on a $\mathrm{MgO}$ crystal (from ref. [3], curve C) and of $\mathrm{YBCO} / \mathrm{N}-\mathrm{YBCO} / \mathrm{YBCO}$ ramp junctions with a non-perovskite $\mathrm{YBCO}$ barrier layer

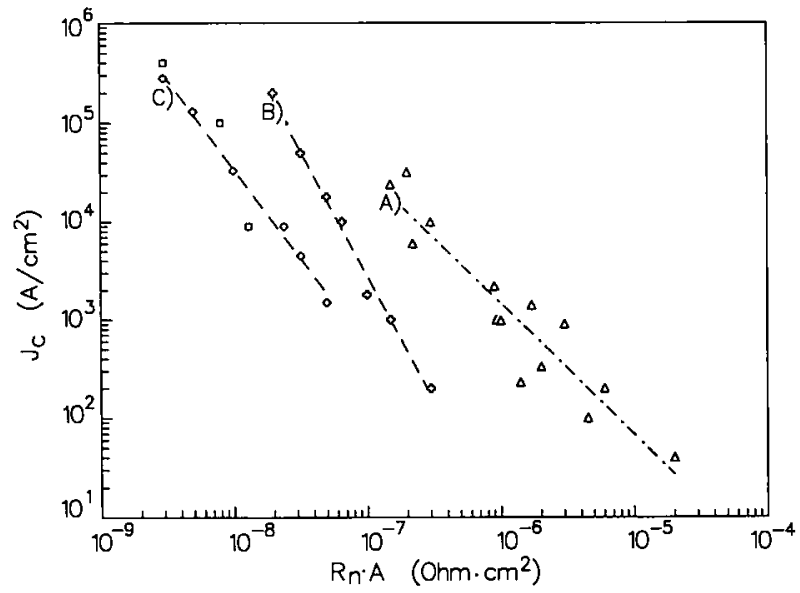

Fig. 2. Critical current density as a function of the specific junction resistance $R_{\mathrm{n}} A$ of the different junctions: YBCO/PBCO/ YBCO junctions (marked by triangle, curve $\mathrm{A}$ ); $\mathrm{GBJ}$ on $\mathrm{SrTiO}_{3}$ bicrystals (from ref. [1], marked by cross, curve B); GBJ on MgO crystal (from ref. [3], marked by square, curve $\mathrm{C}$ ) and $\mathrm{YBCO}$ / $\mathrm{N}-\mathrm{YBCO} / \mathrm{YBCO}$ junctions (from ref. [12], marked by box). 
(from ref. [12], marked by a square). The dependence of $J_{c}$ on the junction specific resistance of our $\mathrm{YBCO} / \mathrm{PBCO} / \mathrm{YBCO}$ junctions is fitted by the curve $J_{\mathrm{c}} \sim\left(R_{\mathrm{n}} A\right)^{-m}$ with $m=1.2$. For SNS structures $J_{\mathrm{c}} \sim\left(R_{\mathrm{n}} A\right)^{-2}$, while for SINS junctions $J_{\mathrm{c}} \sim\left(R_{\mathrm{n}} A\right)^{-1}$ [18]. The observed experimental dependence more closely resembles the SINS structures, while the $J_{\mathrm{c}}\left(R_{\mathrm{n}} A\right)$ dependence of the GBJ and of the junctions with low ohmic natural barriers is close to the theoretical dependence of SNS structures. Also different types of junctions give a similar $J_{\mathrm{c}}$ at different $R_{\mathrm{n}} A$ values. At $J_{\mathrm{c}} \sim 10^{2}-10^{3} \mathrm{~A} / \mathrm{cm}^{2}$, the $R_{\mathrm{n}} A$ values are $\leqslant 10^{-9} \Omega \mathrm{cm}^{2}$ for low ohmic weak link structures, $\sim 10^{-8}-10^{-7} \Omega \mathrm{cm}^{2}$ for GBJ and $10^{-7}-10^{-5}$ $\Omega \mathrm{cm}^{2}$ for YBCO/PBCO/YBCO ramp junctions (see fig. 2). So the nature of the barrier and hence the strength of the Josephson coupling are different in the mentioned types of junctions.

The temperature dependence of the normal state resistance for $\mathrm{YBCO} / \mathrm{PBCO} / \mathrm{YBCO}$ junctions with a thick $\left(L>\xi_{\mathrm{n}}\right)$ barrier is not typical for a simple SNS structure. For SNS structures with $L>\xi_{\mathrm{n}}$ and $L \gg l$ ( $l$ is the mean free path of the carriers), the normal state resistance is governed by the $\mathrm{N}$-layer resistance $R_{\mathrm{p}}$ and the boundary resistance $B_{1}$ and $B_{2}$ :

$R_{\mathrm{n}} \sim R_{\mathrm{p}}+B_{1}+B_{2}$,

where $B_{1}$ and $B_{2}$ denote the boundary resistance between the PBCO barrier and YBCO base and top electrodes, respectively. As we discussed earlier, the boundary resistance is negligible for these structures compared to the barrier resistance $R_{\mathrm{p}}$. Thus, if we suggest $\mathrm{YBCO} / \mathrm{PBCO} / \mathrm{YBCO}$ junctions to be SNS weak link structures, for large thicknesses of the barrier, $L \gg \xi_{\mathrm{n}}, R_{\mathrm{n}}$ must reflect the semiconductor-like temperature dependence $R(T)$ for PBCO [16]. Meanwhile the junction resistance $R_{\mathrm{n}}$ varies insignificantly at low temperatures and different thicknesses of the barrier. Figure 3 shows the junction resistance $R_{\mathrm{n}}$ for $L=8 \mathrm{~nm}$ and $L=20 \mathrm{~nm}$. Typically, $R_{\mathrm{n}}$ decreases with decreasing temperature down to 4 $\mathrm{K}$ for $L<10 \mathrm{~nm}$, in contrast to the junction resistance $R_{\mathrm{n}}(T)$ for $L \geqslant 15-20 \mathrm{~nm}$. However, the total change in the resistance at temperatures $T<T_{\mathrm{c}}$ is negligible in comparison to the $R(T)$ dependence of bulk PBCO material. For different barrier thicknesses the PBCO barrier resistivity $R_{\mathrm{r}} A / L$ is $0.3-0.4$

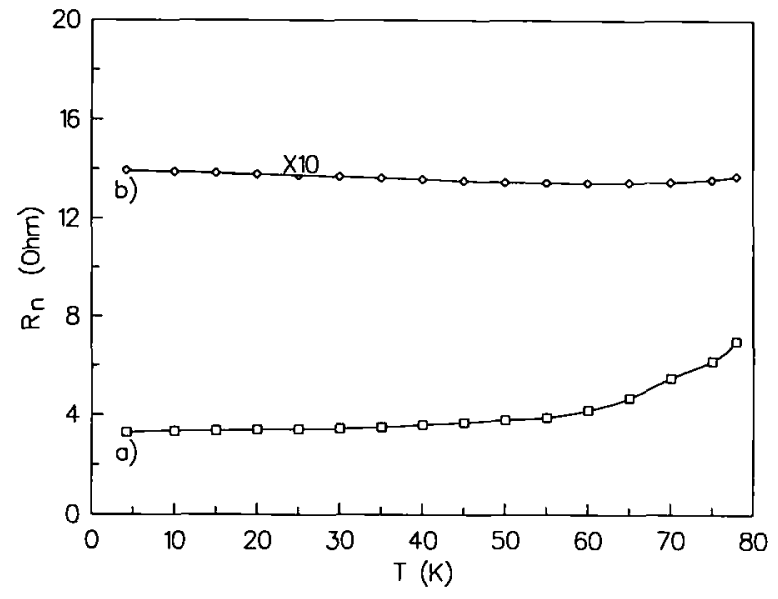

Fig. 3. Temperature dependence of the junction resistance at PBCO barrier thicknesses $L$ : (a) $L=8 \mathrm{~nm}$, (b) $L=20 \mathrm{~nm}$.

$\Omega \mathrm{cm}$ for $L \sim 8-10 \mathrm{~nm}$ and $3-4 \Omega \mathrm{cm}$ for $L \sim 15-20$ $\mathrm{nm}$.

A weak temperature dependence of the junction resistance is more typical for tunnel type junctions and cannot be described by an SNS model. This result, the $J_{\mathrm{c}}\left(R_{\mathrm{n}} A\right)$ dependence and high specific resistance values of our junctions indicate that the Josephson coupling in the $\mathrm{YBCO} / \mathrm{PBCO} / \mathrm{YBCO}$ junctions is established through a high resistive barrier layer. With respect to these characteristics the junctions are better described by a SNINS tunneling model. Nevertheless, some junction characteristics are typical for SNS weak link structures such as the SNS-like $I-V$ curves, the excess current and the temperature dependence of the critical current near $T_{\mathrm{c}}$ [15]. Therefore the junctions studied can hardly be described by simple SNS weak link or SIS tunnel models. Next we will consider some more complex models for directly conducting and tunnel junctions. The comparison with experimental results, especially the dependence of the $I_{\mathrm{c}} R_{\mathrm{n}}$ products on temperature and barrier thickness will be discussed in section 4 .

\section{Models for current passage in the $\mathrm{YBCO} / \mathrm{PBCO} /$ YBCO edge junctions}

\subsection{Proximity effect in the multilayer junctions}

For many proposed SNS structures [1-12] the proximity effect is an intrinsic behavior. Due to the 
small coherence length and low carrier density in HTS and the high sensitivity of the HTS behavior to its oxygen content, two effects are possible for SNS or SIS structures with HTS electrodes. Firstly, the superconducting order parameter in HTS is depressed in the vicinity of the barrier. Secondly, a finite transparency of the SN boundary, which is described as an additional boundary resistance $R_{\mathrm{b}}$, arises between HTS electrodes and the barrier layer. A detailed study of the proximity effect in the "dirty" limit with different boundary conditions has been made by Kupriyanov et al. [18-22]. Their calculations are based on the solution of the Usadel equations with different boundary conditions at the SN boundary in SNS, SNIS or SNINS structures. The proximity coupling and the above-mentioned two effects depend on two dimensionless parameters:

$\gamma=\rho_{\mathrm{s}} \xi_{\mathrm{s}} / \rho_{\mathrm{n}} \xi_{\mathrm{n}}, \quad \gamma_{\mathrm{b}}=R_{\mathrm{b}} / \rho_{\mathrm{n}} \xi_{\mathrm{n}}$,

where $\rho$ and $\xi$ are the resistivity and the coherence length. The indices $s$ and $n$ refer to the $S$ and $\mathrm{N}$ layer, respectively. For SNS structures with $\mathrm{Ag}$ or $\mathrm{Au}$ as a $\mathrm{N}$-layer the smallest estimated parameter $\gamma$ for the coupling in the $a-b$ direction is $\gamma^{\prime \prime} \sim 5$ and for coupling in the $c$-axis direction $\gamma^{\perp} \sim 100$ [19]. An estimate of the carrier scattering at the SN boundary between the YBCO material and the isomorphous normal metal for the case of atomically sharp and "clean" HTS/N boundaries, according to ref. [18], results in:

$$
\begin{aligned}
& R_{\mathrm{b}}^{\perp} \sim 10^{-7} \Omega \mathrm{cm}^{2}, R \sharp \sim 10^{-11} \Omega \mathrm{cm}^{2}, \\
& \gamma_{\mathrm{b}}^{\perp} \sim 10^{4}, \gamma \| 20 .
\end{aligned}
$$

For SNS structures with a low ohmic N-layer the theory mentioned describes the depression of the $I_{\mathrm{c}} R_{\mathrm{n}}$ products and their temperature dependence satisfactorily.

For the $\mathrm{YBCO} / \mathrm{PBCO} / \mathrm{YBCO}$ structures the proximity effect is quite different than in SNS structures with a low ohmic N-layer. Using the experimental data $\rho_{\mathrm{n}} \sim 0.3-3 \Omega \mathrm{cm}, \xi_{\mathrm{n}} \sim 6 \times 10^{-7} \mathrm{~cm}$ and a boundary specific resistance $R_{\mathrm{b}}<10^{-8} \Omega \mathrm{cm}^{2}$ we find

$\gamma^{\prime \prime} \sim 10^{-4}, \quad \gamma_{\mathrm{b}}<10^{-1}$.

So the depression of the superconducting properties of the YBCO layers due to contact with the nonsuperconducting $\mathrm{PBCO}$ layer is negligible from the es- timation of the proximity effect parameters. The estimate using eq. (3) strongly depends on the parameters used for the N-layer. We can suggest the existence of an $(\mathrm{Y}, \mathrm{Pr}) \mathrm{BCO}$ layer between the $\mathrm{YBCO}$ and PBCO layers. However, the boundary conditions are similar in the junctions with different barrier thicknesses. Thus, such an interdiffusion effect must be similar in all junctions and cannot explain the dependence of the junction parameters on the PBCO barrier thickness.

The analysis of the proximity effect in HTS SNS structures has been made also by Deutscher and Simon using De Gennes boundary conditions [23]. Their conclusion about the advantage of the coupling through a low carrier density semiconductor layer agrees with this estimate of the proximity effect parameters for $\mathrm{YBCO} / \mathrm{PBCO} / \mathrm{YBCO}$ structures.

\subsection{Models with localized electronic states in the barrier}

The presence of defect levels in the semiconducting or insulating barrier can facilitate the current passage between the superconducting electrodes. Depending on the thickness and the nature of the barrier, the concentration and location of impurities in the barrier - at the interface, in the middle of the barrier or having a periodic distribution across the barrier - different mechanisms of the resonant current passage can be realized [24-28].

In Halbritter's formulation [24] the localized states in an oxide layer adjacent to the metal electrodes hybridize with conclusion carriers forming interface states of different types. These states can change significantly the junction resistance at low voltage and weaken the supercurrent of the junction. This model gives the following $J_{\mathrm{c}} \rho_{\mathrm{n}}$ dependence on the barrier thickness $L$ [25]:

$J_{\mathrm{c}} \rho_{\mathrm{n}} \sim \exp (-a L), \quad J_{\mathrm{c}} \sim \rho_{\mathrm{n}}^{-2}$,

which is close to the experimentally observed dependence in the grain boundary junctions. Meanwhile, this model suggests a strong (exponential) dependence of the resonant tunneling current on the voltage (in the low voltage range $V \leqslant V^{*} \sim 5-20 \mathrm{mV}$ ) and on the temperature. A similar temperature dependence is predicted for resonant tunneling via periodically arranged localized states in the barrier, ac- 
cording to refs. [26] and [27]. Such periodically distributed impurities form a narrow impurity band with a width [26]

$B=\pi T \ln \left[\left(E_{\mathrm{c}}-E_{\mathrm{F}}\right) /\left|\left[\ln \left(c a_{\mathrm{B}}^{3}\right)\right]\right|\right.$,

changing the dependence of the junction parameters on the barrier thickness [27]. In eq. (7) the barrier height is $E_{\mathrm{c}}-E_{\mathrm{F}}, c$ is the density of the localized states, and $a_{\mathrm{B}}$ is the Bohr radius of the impurity.

The carriers can tunnel via impurity levels by inelastic scattering. This channel causes a nonsuperconducting current via the barrier changing the junction normal state resistance $[24,28]$. For a channel with $\mathrm{n}$-impurity atoms the proper contributions is [28]

$\sigma_{\mathrm{n}} \sim T^{n-[2 /(n+1)]} \exp \left[-2 L /(n+1) a_{\mathrm{B}}\right]$.

In the tunnel junctions with impurity levels in the barrier the tunnel mechanism of current flow is effective only at very low temperatures. An increase in temperature activates inelastic channels characterized by a larger number of impurities. As can be seen from eq. (8), a temperature independent contribution is ensured only by an inelastic tunneling channel through one impurity center.

\subsection{Models with strong pair breaking states in the barrier}

In the models described in section 3.2. the conduction carriers interact with localized nonmagnetic impurity levels in the barrier or at the interface. If the localized states have a magnetic moment or if another strong pair breaking mechanisms is in effect, there is an additional strong destruction of the superconducting junction parameters. This pair breaking can be described by introducing the characteristic time parameter $\tau^{*}$ for electron scattering accompanied by spin reversal $[29,30]$. The effect of magnetic pair breaking on the $I_{\mathrm{c}} R_{\mathrm{n}}$ product of SNS structures with transparent interface (low proximity effect parameters $\gamma \ll 1$ and $\gamma_{\mathrm{b}} \ll 1$ ) has been analyzed in ref. [18]. For SNINS structures with similar interface conditions this effect has been analyzed in ref. [22]. The strong pair breaking centers in the $\mathrm{YBCO} / \mathrm{PBCO} / \mathrm{YBCO}$ junctions may be the Pr ions. In some types of HTS junctions the scattering and pair breaking may also be due to the localized uncompensated spin magnetic moments of $\mathrm{Cu}^{2+}$ in the oxygen depleted barrier or in the near-barrier region of HTS junctions.

\section{Discussion of the models and experimental results}

The low conductivity of the PBCO barrier layer in comparison with the YBCO layers leads to small proximity effect parameters (see eq. (5)). The boundary conditions are similar in all examined junctions and do not affect strongly the barrier thickness dependence of the junction parameters. So the junction characteristics can be treated as being governed by the barrier thickness and its nature. A small depression of the superconducting parameters at the interface between the layers and a low boundary resistance are important features of the $\mathrm{YBCO} / \mathrm{PBCO} /$ YBCO ramp junctions.

Figure 4 shows the dependence of the $I_{\mathrm{c}} R_{\mathrm{n}}$ products on the PBCO barrier thickness. The experimental data are given by the squares. The solid line (a) is a fitting curve. Curves (1) and (2) are theoretical $I_{\mathrm{c}} R_{\mathrm{n}}$ dependences for $\gamma \ll 1$ and for different values of the $\xi_{n}: 8 \mathrm{~nm}$ (curve 1) and $5 \mathrm{~nm}$ (curve 2). Better fitting is possible only at lower values of the coherence length $\xi_{n} \leqslant 1-2 \mathrm{~nm}$, which is consistent with the

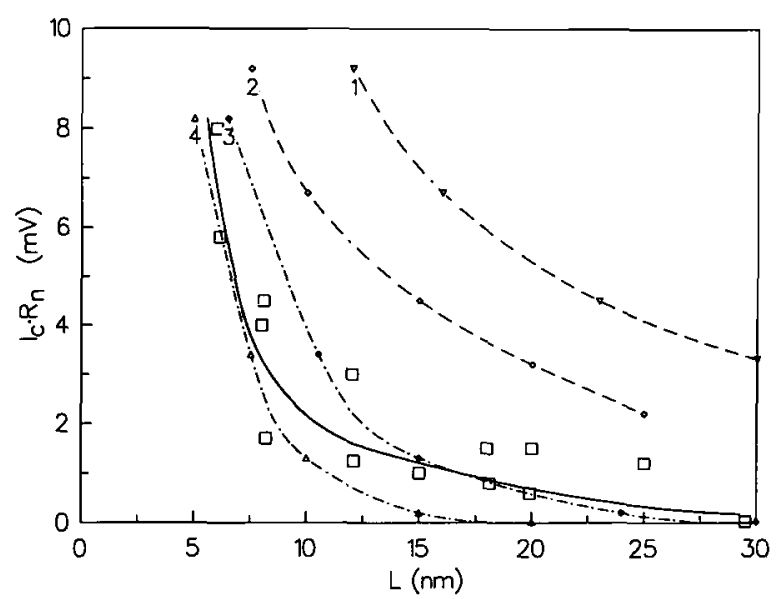

Fig. 4. Dependence of the $I_{\mathrm{c}} R_{\mathrm{n}}$ products on the barrier thickness. Experimental data are marked by a box, fitted by a solid curve. Calculated $I_{\mathrm{c}} R_{\mathrm{n}}(L)$ dependences: the SNINS structure with $\gamma \ll 1$ and $\xi_{n}: 8 \mathrm{~nm}$ (curve 1 ) and $5 \mathrm{~nm}$ (curve 2); the SNINS structure with $\gamma \ll 1, \tau^{*}=1 / \pi T_{\mathrm{c}}$ and $\xi_{\mathrm{n}}: 7 \mathrm{~nm}$ (curve 3 ) and $5 \mathrm{~nm}$ (curve 4). 
$J_{\mathrm{c}}(L)$ dependence for barrier thicknesses $L \geqslant 10 \mathrm{~nm}$.

Two typical dependences of the $I_{\mathrm{c}} R_{\mathrm{n}}$ products on temperature are shown in fig. 5 as curves (a) and (b) (solid lines). For curve (a) we try to find the fitting curve by using the proximity effect theories [19,22]. Using $I_{\mathrm{c}} R_{\mathrm{n}}$ dependences from ref. [19] for SNS structures for $L / \xi_{n}=2$ and large values of $\gamma_{\mathrm{b}}$ we obtain curve 1 (dotted line). A large value of $\gamma_{b}$ is needed to explain the $I_{\mathrm{c}} R_{\mathrm{n}}$ value at $T=4 \mathrm{~K}$. The discrepancy with the experimental data (curve a) is large at all temperatures. Using the $I_{\mathrm{c}} R_{\mathrm{n}}(T)$ dependence of SNINS structures for $L / \xi_{n}=2$ and small values of $\gamma_{b}$ (dash-dotted line), the discrepancy is reduced especially at low temperatures but the agreement is still not satisfactory. The calculated $I_{\mathrm{c}} R_{\mathrm{n}}(T)$ dependences for SNS or SNINS structures with a lowered $I_{\mathrm{c}} R_{\mathrm{n}}$ product due to the proximity effect have a shape, which is different from the experimental ones. These results and the high critical temperature $T_{\mathrm{co}}$ of the examined junctions also contradict the possible interdiffusion of $\mathrm{Y}$ and $\mathrm{Pr}$ atoms and formation of the SNINS structure with $(\mathrm{Y}, \mathrm{Pr}) \mathrm{BCON}$ layer.

YBCO/PBCO/YBCO junctions posses a high specific resistance of the barrier $R_{\mathrm{n}} A \sim 10^{-7}-10^{-5} \Omega \mathrm{cm}^{2}$. For these resistance values the contribution of the

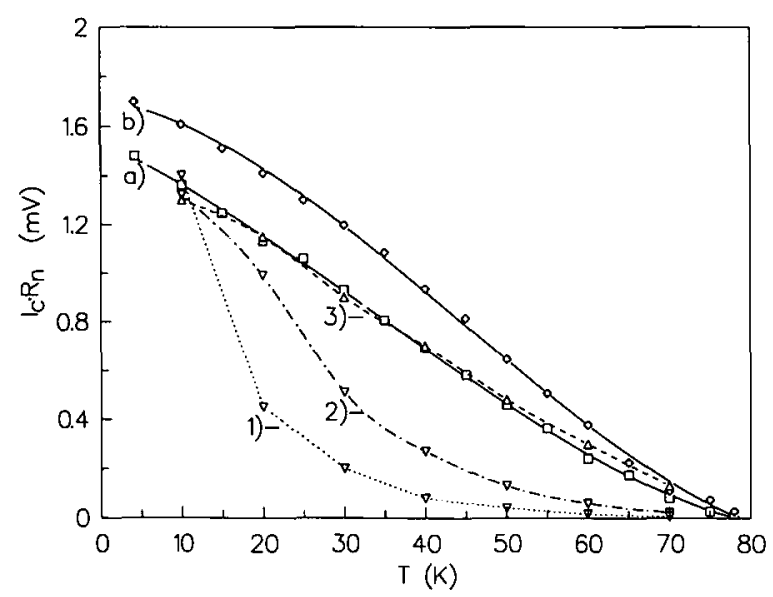

Fig. 5. Temperature dependences of the $I_{\mathrm{c}} R_{\mathrm{n}}$ products of the junctions with PBCO barrier thicknesses: (a) $20 \mathrm{~nm}$; (b) $8 \mathrm{~nm}$. Experimental data are fitted by the solid lines. Curves $1-3$ are calculated dependences for: (1) the SNS structure with $L / \xi_{n}=2$ and large $\gamma_{\mathrm{b}}$ value; (2) the SNINS structure with $L / \xi_{\mathrm{n}}=2$ and $\gamma \ll \mathrm{I}$ : (3) the SNINS structure with $L / \xi_{\mathrm{n}}=1.5, \gamma \ll 1$ and pair breaking parameter $\tau^{*}=1 / \pi T_{\mathrm{c}}$. resonant tunneling due to the defects in the barrier crystal structure is more effective than in low ohmic junctions. Nevertheless the resonant supercurrent passage gives a strong temperature and voltage dependence of the junction parameters. As shown in figs. 3 and $5, R_{\mathrm{n}}$ was fairly temperature independent and $J_{\mathrm{c}}$ does not change exponentially with temperature. The $I-V$ characteristics do not contain lowvoltage anomalies, typical for resonant current tunneling models. Also the $J_{\mathrm{c}}\left(R_{\mathrm{n}} A\right)$ dependence differs from that calculated by eq. (6). So the contribution of the resonant supercurrent passage seems to be small in the examined junctions.

The current passage with inelastic scattering on one effective impurity center seems to be possible in the studied structures with $\mathrm{PBCO}$ barrier thicknesses $L \sim 8-20 \mathrm{~nm}$. This mechanisms and the tunnel mechanisms give a temperature independent contribution to the junction conductivity. This contribution can be due to the specific electrical transport behavior of the PBCO material which is different from the other superconducting $1: 2: 3$ compounds with a metallic-like resistance $R(T)$. Nevertheless, an inelastic scattering mechanism cannot explain the observed dependence of the $I_{\mathrm{c}} R_{\mathrm{n}}$ products on temperature and the fast decrease of the $I_{\mathrm{c}} R_{\mathrm{n}}$ product with barrier thickness.

A sufficiently good description of the $I_{\mathrm{c}} R_{\mathrm{n}}(T)$ dependence and the values of the $I_{\mathrm{c}} R_{\mathrm{n}}$ product at different barrier thicknesses is obtained by taking into account the carrier scattering at the localized pair breaking centers in the barrier. We found good agreement with the experiment data using a model for SNINS structures with magnetic pair breaking in the barrier [22]. The calculated $I_{\mathrm{c}} R_{\mathrm{n}}(T)$ dependence for SNINS structures with $L / \xi_{n}=1.5, \gamma_{\mathrm{b}} \ll 1$ and a temperature independent pair breaking parameter $\tau^{*}=1 / \pi T_{\mathrm{c}}$ is shown in fig. 5 as curve 3 , dashed line. In addition, the calculated $I_{\mathrm{c}} R_{\mathrm{n}}(L)$ dependence for $\xi_{n}=7 \mathrm{~nm}, \gamma \ll 1$ and $\tau^{*}=1 / \pi T_{\mathrm{c}}$ (fig. 4 , curve 3 ) fits the experimental data better than the other models. Thus, the pair breaking mechanism gives a strong temperature independent suppression of the $I_{\mathrm{c}} R_{\mathrm{n}}$ products which may explain our experimental data of $I_{\mathrm{c}} R_{\mathrm{n}}(T)$ and $I_{\mathrm{c}} R_{\mathrm{n}}(L)$.

Both effects - inelastic scattering and strong pair breaking in $\mathrm{YBCO} / \mathrm{PBCO} / \mathrm{YBCO}$ junctions - are due to the specific PBCO magnetic and electric 
transport properties. For a further analysis and a quantitative description of the junction behavior more information about the effect of $\operatorname{Pr}$ on the behavior of $\mathrm{PBCO}$ is needed. The electrical transport and magnetic properties of the (Y, Pr)BCO compound and the YBCO/PBCO superlattices are studied intensively now, but the results are partially contradictory and not conclusive. Further study is necessary, especially with respect to the electrical transport mechanisms in HTS and related perovskite structures, as well as the nature of the pair breaking effect in the $(\mathrm{Y}, \mathrm{Pr}) \mathrm{BCO}$ compound, $\mathrm{YBCO} / \mathrm{PBCO}$ superlattices and $\mathrm{YBCO} / \mathrm{PBCO} /$ YBCO Josephson junctions.

The transport properties of the grain boundary junctions have been explained in ref. [25] by a model based on a insulating barrier at the grain boundary interface containing a large number of localized defects. The tunnel current passage in the junctions with a PBCO barrier is to be preferred if we compare the junction specific resistances of the grain boundary junctions and $\mathrm{YBCO} / \mathrm{PBCO} / \mathrm{YBCO}$ ramp junctions (fig. 2). The effect of the defect impurity levels in the barrier may be also different. In the GBJ these defects are created by a disordered high angle grain boundary, while in the YBCO/PBCO/YBCO ramp junctions the current is established by the passage through the $\mathrm{PBCO}$ layer. The interfaces between $\mathrm{YBCO}$ and $\mathrm{PBCO}$ layers weakly affect the $\mathrm{YBCO} /$ $\mathrm{PBCO} / \mathrm{YBCO}$ junction characteristics.

\section{Summary}

The present study of the YBCO/PBCO/YBCO junctions with and without $\mathrm{PBCO}$ barrier shows that the characteristics of these structures are determined by the thickness of the PBCO barrier and its nature. The boundary resistance $R_{\mathrm{b}}$ and the depression of the YBCO superconducting parameters near the interface are negligible for the fabrication process used and do not affect strongly the junction behavior. Different models of the reduction of the $I_{\mathrm{c}} R_{\mathrm{n}}$ product are discussed to describe the $\mathrm{YBCO} / \mathrm{PBCO} / \mathrm{YBCO}$ junctions characteristics. The dependence of the $I_{\mathrm{c}} R_{\mathrm{n}}$ products on barrier thickness and temperature differs from the expected dependences of SNS or SNINS structures with strong proximity effect. The resonant tunneling via impurity levels cannot give a large contribution to the junction supercurrent $J_{\mathrm{c}}$ for the PBCO barrier thicknesses used. Nevertheless, a contribution to the junction conductivity by resonant tunneling with inelastic scattering at one impurity center cannot be excluded. Good agreement with the experimental dependence of the $I_{\mathrm{c}} R_{\mathrm{n}}$ product on temperature and barrier thickness is obtained if a strong pair breaking mechanism in the barrier is taken into account. The effect of praseodymium on the electric transport in the PBCO material needs further study.

\section{Acknowledgements}

The authors are grateful to A.A. Golubov, B.B.G. Klopman and M.V. Pedyash for the discussion of the results. We acknowledge the helpful discussion and assistance of our collegeas at the Low Temperature Laboratory. This work was supported by the Dutch National Research Program on High- $T_{\mathrm{c}}$ Superconductivity, the Netherlands Technology Foundation and the Philips Corporation.

\section{References}

[1] R. Gross, P. Chaudhari, M. Kawasaki and A. Gupta, Rhys. Rev. B 42 (1990) 10735.

[2] K. Char, M.S. Colclough, L.P. Lee and G. Zaharchuk, Appl. Phys. Lett. 59 (1991) 2177.

[3] S.E. Russek, D.K. Lathrop, B.H. Moeckly, R.A. Buhrman, D.H. Shin and J. Silcox, Appl. Phys. Lett. 57 (1991) 1155.

[4] K.P. Daly, W.D. Dozier, J.F. Burch, S.B. Coons, R. Hu, C.E. Platt and P.W. Simon, Appl. Phys. Lett. 58 (1991) 543.

[5] M.G. Forrester, J. Talvacchio, J.R. Gavaler, M. Rooks and J.L. Lindquist, IEEE Trans. Magn. 27 (1991) 3098.

[6] M.S. Dilorio, S. Yoshizumi, K.-Y. Yang, J. Zang and M. Maung, Appl. Phys. Lett. 58 (1991) 2552.

[7] K.H. Ono, J.A. Beal, M.W. Cromar, in: Ext. Abstract of ISEC"91 Int. Conf., Glasgow, UK (1991) 386.

[8] D.K. Chin and T. van Duzer, Appl. Phys. Lett. 58 (1991) 753.

[9] J.B. Barner, C.T. Rogers, A. Inam, R. Ramesh and S. Bersey, Appl. Phys. Lett. 59 (1991) 742.

[10] J. Gao, W.A.M. Aarnink, G.J. Gerritsma and H. Rogalla, Physica C 171 (1990) 126.

[11] G. Koren, E. Polturak, E. Aharoni and D. Cohen, Appl. Phys. Lett. 59 (1991) 2745.

[12] B.D. Hunt, M.C. Foote and L.J. Bajuk, Appl. Phys. Lett. 59 (1991) 982 . 
[13] J. Gao, W.A.M. Aarnink, G.J. Gerritsma, A.J.H.M. Rijnders, H. Rogalla, F. Hakkens, W. Coene and M.A.M. Gijs, Physica C 177 (1991) 384.

[14] J. Gao, Yu.M. Boguslavskij, B.B.G. Klopman, D. Terpstra, G.J. Gerritsma and H. Rogalla, Appl. Phys. Lett. 59 (1991) 2754.

[15] J. Gao, Yu.M. Boguslavskij, B.B.G. Klopman, D. Terpstra, G.J. Gerritsma and H. Rogalla, J. Appl. Phys. (1991), submitted.

[16] K.K. Likharev, Rev. Mod. Phys. 51 (1979) 116.

[17] M.E. Lopez-Moralez, D. Rios-Jara, J. Taquena, R. Escudero and S. La Placa, Phys. Rev. B 41 (1991) 6665.

[18] M.Yu. Kupriyanov and K.K. Likharev, Usp. Fiz. Nauk. 160 (1990) 49 (Sov. Phys. Usp. 33 (1990) 340).

[19] M.Yu. Kupriyanov and K.K. Likharev, IEEE Trans. Magn. 27 (1991) 2460.

[20] M.Yu. Kupriyanov and V.F. Lukichev, Zh. Eksp. Teor. Fiz. 94 ( 1988 ) 138 (Sov. Phys. JETP 67 (1988) 1163).
[21] A.A. Golubov and M.Yu. Kupriyanov, Zh. Eksp. Teor. Fiz. 96 (1989) 1420 (Sov. Phys. JETP 69 (1988) 805).

[22] A.A. Golubov and M.Yu. Kupriyanov, Physics Lett. A 154 (1991) 181 .

[23] G. Deutscher and R.W. Simon, J. Appl. Phys. 69 (1991) 4137.

[24] J. Halbritter, J. Appl. Phys. 58 (1985) 1320.

[25] R. Gross and B. Mayer, Physica C 180 (1991) 235.

[26] L.G. Aslamazov and M.V. Fistul', Zh. Eksp. Teor. Fiz. 83 (1982) 1720 (Sov. Phys. JETP 56 (1982) 666).

[27] A.V. Tartakovskij and M.V. Fistul', Zh. Eksp. Teor. Fiz. 94 (1988) 353 (Sov. Phys. JETP 67 (1988) 1935).

[28] L.I. Glazman and K.A. Matveev, Zh. Eksp. Teor. Fiz. 94 (1988) 332 (Sov. Phys. JETP 67 (1988) 1276).

[29] A.A. Abrikosov and L.P. Gor'kov, Zh. Eksp. Teor. Fiz. 39 (1960) 1781 (Sov. Phys. JETP 12 (1961) 1243).

[30] P.G. De Gennes, Superconductivity of Metals and Alloys, 2nd ed., (Addison-Wesley, New York, 1988). 